\title{
Computer Simulation of Turbulent Flow Phenomena in Nuclear Fuel Pin Subassemblies
}

\author{
Hisashi Ninokata and Elia Merzari \\ Nuclear Engineering Department, Tokyo Institute of Technology \\ N1-5, 2-12-1 O-okayama, Meguro-Ku, Tokyo 152-8550 Japan \\ e-mail: hninokat@nr.titech.ac.jp
}

\begin{abstract}
This paper focuses on the turbulence phenomena that have been identified by computational fluid dynamics (CFD), and that are peculiar to the flows in tight lattice nuclear fuel subassemblies, eccentric annuli and two-rectangular channels interconnected by a narrow gap. The CFD methods employed are the Direct Numerical Simulation of turbulence (DNS) and Large Eddy Simulation (LES) techniques. Highlighted phenomena include turbulence-driven secondary flows inside a subchannel, local turbulent-laminar transition in the narrow gap region between two adjacent fuel pins, and global pulsation of the flow across the length of a fuel subassembly. Special attention will be paid on linkage to the physics, limitation of the computational capability of the CFD methods, and the usefulness of the technology in reference to the foreseeable future.
\end{abstract}

Keywords : DNS, LES, Secondary flow, Laminar-turbulent transition, Global pulsation

\section{Introduction \\ 1.1 Computational fluid dynamics}

Fluid dynamics has a long history and is an established technical discipline being backed-up with deep theoretical contributions and recent sophisticated computational techniques. With the enormous and rapid development in computing power and resources in these decades, it is not surprising to hear optimistic visions that the time will come when the Computational Fluid Dynamics CFD can produce data of equivalent high quality to experiment, thus replacing almost entirely high-cost large scale mockups or any scaled experiments. In the paragraphs that follow, however, we will be based on reality and show what the most advanced CFD can achieve on one of the high-end computers available in the world. Focus will be placed on several aspects of CFD relevant to the nuclear fuel pin subassembly thermal hydraulics.

There should be no questions that the NavierStokes (N-S) equations can describe adequately the detailed motion of coolant and the forces exerted onto the fuel pin wall surfaces and the surrounding structure. It is believed that, if we solve directly the N$\mathrm{S}$ equations without bringing in any modeling or approximations, we can capture fluid phenomena with higher resolution than that our measurement technology can provide. Obviously this is the approach of direct numerical solution or simulation (DNS) of turbulence. Unfortunately the DNS requires prohibitively larger memory and longer CPU running time than even the most high-end computers to date can provide. Therefore, without sufficient computing power in most cases, major efforts in fluid dynamics involve a wide variety of assumptions and numerical approximations to make solving the N-S or related equations tractable. Such coolant simulations are at the heart of CFD based on the RANS (ReynoldsAveraged Navier-Stokes equations.
Flows of interest in this paper are characterized by unsteady and irregular flows which give something of the appearance of randomness; strong vorticity; stirring and diffusion of heat; dissipation of energy by momentum exchange. In fully developed turbulent flows in a fuel pin subassembly, there are a couple of interesting phenomena that have never been captured by traditional RANS-CFD. Instead, they had been found theoretically or experimentally; and a-posteriori calculations were carried out to reproduce or investigate the mechanisms. They include turbulencedriven secondary flows in subchannels, local transition between laminar and turbulent flows near a narrow gap between two adjacent fuel pins in particular in the case of tight lattice fuel pin subassemblies, and the global pulsations leading to the coolant mixing between two subchannels. These particular phenomena are the subject of DNS and LES in this article and discussed in the following Sections 2 and 3.

\subsection{Computing power required by DNS}

In order to delineate dynamics of the coolant flows by DNS, the use of mesh grids fine enough to capture the smallest turbulent eddies is necessary. The smallest eddy length scale normally refers to as the Kolmogorov length scale $\eta$. The mesh size ( $\Delta$ ) requirement in this case is $\pi \eta / \Delta>1.5$. The rough estimate of the number of computational grids per unit volume would be in proportion to $\mathrm{Re}^{9 / 4}$ whereas Large Eddy Simulation (LES) requires those in proportion to $\mathrm{Re}^{5 / 3}$. Actual numbers of gird points used in our work using the Earth Simulator ${ }^{1)}$ are illustrated in Figure 1. Here, the Earth Simulator is one of the most powerful high-end TeraFLOPS machines available in the world.

At the same time the computational domain should be expanded enough to include a reasonable volume of the fuel pin assembly. Note that even if a time averaged distribution is spatially symmetric, an 
instantaneous snapshot is not symmetric. Therefore DNS cannot take the advantage of this symmetry in defining the computing domain. These result in a requirement well beyond current computational capabilities available in the world, although it may be possible in the future. Some experts, however, say that this is very far distant even with the most optimistic estimate of the growth in computing power. In fact, using the Earth Simulator, the DNS calculation of a fully developed turbulent flow of $\operatorname{Re} \sim 2.3 \times 10^{4}$ in an eccentric annulus channel took almost 6 months to attain a convergence with $1.36 \times 10^{8}$ computing meshes. This will be illustrated in Section 3 in this article. Here we will not attempt to review the multitude of approaches of CFD, e.g., DNS, subgrid-scale modeling of Large Eddy Simulation (LES), and turbulence modeling of higher to lower degrees for Reynolds Averaged N-S (RANS) equations, but merely make a brief observation.

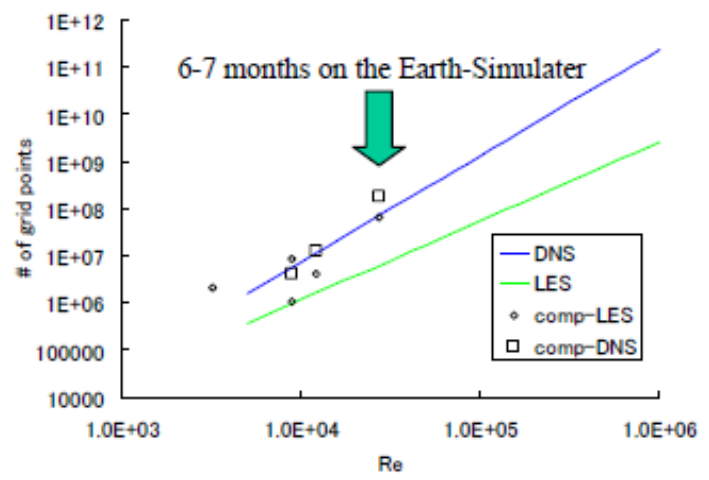

Figure 1. Rough estimate of the required number of mesh grid points for DNS and LES: theoretical (solid lines) and actual on the Earth Simulator (round and square dots).

\subsection{Simulation-based design approach}

The simulation-based design approach consists of computational and experimental approaches. In most cases, both approaches depend on each other and cannot be separated. It is underlined here that only a tiny fraction of the simulations generated in nuclear industries are done using CFD, indicating more space for CFD to come into play. This is not because the CFD is not reliable but designing a nuclear reactor requires well-established and well-proven technology with the sufficiently long term expertise accumulated in the past. Therefore, the full use of CFD in the simulation-based design procedure would be a challenge in the future nuclear industry. It is indeed our challenge to make the CFD's contributions greater in the simulation-based design approach. The remainders continue to be done with physical models in small- to medium-scale similitude experiments and in separate effects experiments. Full mock-up experiments may be requested whenever necessary.

Under these circumstances, the CFD that requires turbulence models provides limited insights into physics because the constitutive equations or closure relations in the RANS equation system require detailed turbulence information from experiment or from DNS. Namely, the RANS is very useful only when mechanisms of the phenomena are known and well understood. This would not change in the future. While experiments provide the information, however, CFD including LES and DNS can provide us more detailed information than experiment for better understanding the phenomena that are observed in the experiment. In this sense, CFD and experiments are always complementary. With well understood phenomena and their modeling, large scale mock up experiments could be replaced partially by CFD, and this would be one of the current targets of the simulation-based design approach.

Finally, the various approaches to CFD result in various computational requirements; yet there is no universal approach other than directly solving the N-S equations (DNS), computational results are substantially dependent on the user specified mesh schemes and boundary conditions, numerical schemes in general, modeling selections which require the user knowledge on turbulence and expertise in creating specific simulation models and in interpreting the results of the simulations.

\section{Fluid Dynamics Phenomena in a Tight Lattice Fuel Pin Subassembly}

When thermal hydraulics in actual nuclear fuel subassemblies (i.e., rod bundles) is concerned, especially when fuels are wire-wrapped, the use of CFD has been limited. Instead, subchannel analysis has been most commonly used with appropriate wire spacer modeling ${ }^{2)}$. In this Section, our discussions will be restricted to the subassemblies consisting of straight bare fuel pins without spacers. This is a first step to look into a detail of the physics and delineate what is happening in the subassemblies.

In the past, the direct numerical simulation (DNS) has hardly been applied to the turbulent flows inside a fuel pin subassembly because of the computing power available at that time. Therefore numerical estimation of detailed thermal hydraulics fields in fuel subassemblies has been mainly carried out by the RANS approach (for example ${ }^{3-5)}$ ). In general, however, it is often the case for the most popular and classical k- $\varepsilon$ turbulence models to fail in predicting turbulence-driven secondary flows, which are caused by the turbulence anisotropy. Furthermore the use of the wall function model substantially limits its applicability to these flows.

In the following, two typical turbulent flow phenomena are shown which the isotropic turbulence models are not capable of predicting: i.e., the turbulence-driven secondary flow that gives significant influences on the main axial flow velocity and wall shear stresses distributions, and the global pulsation phenomena over a fuel axial span of several wave lengths, at least, of the oscillation. The local transition between turbulent and laminar flows is also 
observed in both examples but this will be discussed in Section 3 in more details.

\subsection{DNS calculation of the turbulent flows in a tight-lattice subassembly \\ 2.1.1 Outline of the DNS algorithm}

The governing Navier-Stokes equations, extended to the boundary-fitted coordinate system, are the mass and momentum conservation equations and a mean axial pressure gradient is an input that drives incompressible flows through the flow channel. An attempt was made to minimize the errors arising from this coordinate transformation by adopting the fine meshes that are strictly orthogonal near the wall region and as much orthogonal as possible over the whole domain. The numerical method is based on the fractional time step method ${ }^{6}$, with the collocated grid system. For the spatial derivatives, a second order accurate central difference scheme is applied to the convection and diffusion terms. An explicit AdamsBashforth scheme is used for the time-advancement of convection and diffusion terms. The Poisson-type pressure equation is discretized to yield a large and sparse 7-striped matrix and solved by an iterative plane-by-plane method. Briefly, in each iterative step, assuming a periodic boundary condition in the main stream direction, an FFT solver is applied to the axial direction and the matrix to be solved is reduced to a 5stiped matrix equation, which is solved by a scaled conjugate gradient method. The contravariant velocity components are transformed from the Cartesian velocity components by 4-point interpolation. More detailed description of the method is found in Misawa, et al., ${ }^{\text {) }}$

\subsubsection{Computational domain, boundary conditions and compromises}

DNS calculations in this section focus on an infinite triangular array of the fuel pin subassemblies. A fraction of the infinite array bare pin subassembly is shown in Figure 2(a). This subassembly can be divided into minimum unit cells taking advantage of a maximum symmetry of a subchannel. In this case one minimum unit cell is $1 / 6$ of the subchannel. Note that this unit cell could be a minimum computational domain for RANS but not for DNS because in DNS the instantaneous flow field is not symmetric while its time average is.

As a consequence, for a triangular pin configuration, we need to employ the computational domain that consists of 12 minimum unit cells as illustrated in Figure 2(b), in which the periodic condition is applied at the boundary surfaces that face each other across the fuel pin. The non-slip condition is imposed on the fuel wall surface, and a periodic condition is imposed at the flow inlet and outlet with the axial spacing of $3.2 \times D_{h}$, where $D_{h}$ is the equivalent hydraulic diameter.

The fully-developed turbulent flow is identified by the fact that the sample data of the averaged turbulent energy, bulk velocity, and wall shear stress show statistically a steady-state. The instantaneous velocities obtained by the present simulation are timeaveraged and space-averaged in the axial direction. Those are also ensemble-averaged over all minimum unit cells, and are described using cylindrical coordinate system as shown in Figure 2(c).

Simulations of the flows of friction Reynolds number $\mathrm{Re}_{\tau}=400$ and 600 have been carried out for the pitch-to-diameter ratio $\mathrm{P} / \mathrm{D}=1.2,1.1$ and 1.05, and the flows of $\mathrm{Re}_{\tau}=1,000$ and 1,400 for $\mathrm{P} / \mathrm{D}=1.2$. Here friction Reynolds number $\operatorname{Re}_{\tau}$ is defined by $\bar{w}_{\tau} D_{h} / v$ where $\bar{w}_{\tau}\left(\equiv \sqrt{\overline{\tau_{w}} / \rho}\right)$ is the friction velocity and $\overline{\tau_{w}}$ is the averaged wall shear stress over the wall surface, respectively; and $v$ is the kinematic viscosity. Table I (attached after the list of references) summarizes the computational cases. Case D is with $\mathrm{P} / \mathrm{D}=1.2$ compared to the experiment by Trupp, et al., $\left.{ }^{8}\right)$ having the bulk Reynolds number $\left(\mathrm{Re}_{\text {bulk }}\right)$ of 23,760 which is defined by $w_{b} D_{h} / v$ where $w_{b}$ is the bulk velocity in the subchannel. This experimental result corresponds to the lowest bulk Reynolds number data in a triangular pin bundle and is one of the few in open literature in which details of turbulent characteristics are available.

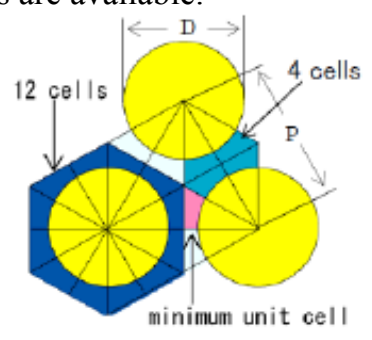

(a) Unit cell arrangement

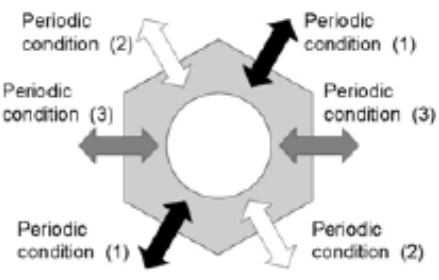

(b) 12 cell DNS domain

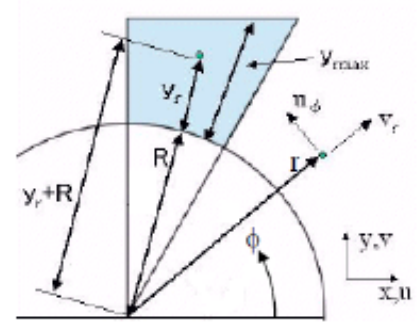

(c) Cylindrical coordinate system

Figure 2. Computational domain, boundary condition and definition of cylindrical coordinate system 


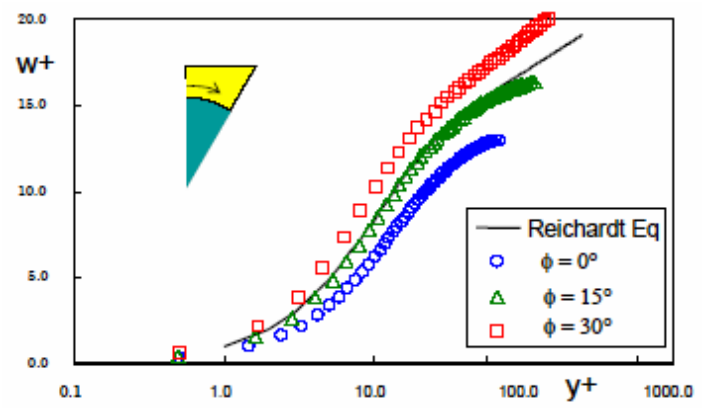

Figure 3. Comparison of axial flow distribution between DNS and a typical log-law wall function $\mathrm{P} / \mathrm{D}=1.05 ; \mathrm{Re}_{\text {bulk }}=9,500$

For example, Figure 3 illustrates a comparison of axial flow distribution between DNS case B1 and a typical log-law wall function model $\left(\mathrm{P} / \mathrm{D}=1.05 ; \mathrm{Re}_{\text {bulk }}=9,200\right)$. All of three plot lines at different angle positions $(\phi=0$, $15,30^{\circ}$ ) are in good agreement with the wall function model if normalized by the local wall shear velocity. In general, comparisons of our DNS results with experiment and theory show satisfactory agreement, indicating the approach could be successfully carried on and, thanks to the continuous growing of available computing power, could become a more practical tool.

\subsubsection{Influence of Reynolds number on turbulent flow fields}

Local transition of laminar-turbulence DNS calculations for the lower bulk Re numbers $\left(\operatorname{Re}_{\text {bulk }}\right)$ for $\mathrm{P} / \mathrm{D}=1.2$ have shown that the distribution shape of Reynolds normal and shear stresses varies from the gap to open region, i.e., from $\phi=0^{\circ}$ to $30^{\circ}$ while, for the higher $\operatorname{Re}_{\text {bulk }}$ number $(>20,000)$ flows, its dependency on the location is found weak. Computational results in a range of $\mathrm{Re}_{\text {bulk }}$ from 4,000 to 20,000 show that a turbulent region is developing into the narrow gap region as $\mathrm{Re}_{\text {bulk }}$ increases but still a locally laminar flow region exists. In this regard, this range is considered to be the one with a local transition of laminar to turbulent flow. DNS has shown the flow is fully turbulent with $\mathrm{Re}_{\text {bulk }} \sim 24,000$. Without solid experimental evidences, however, it is our inference that, for flows in fuel pin subassemblies, the localized turbulent-laminar transition is taking place with the lower $\mathrm{Re}_{\text {bulk }}$ than $\sim 20,000$ and this Reynolds number would change as $\mathrm{P} / \mathrm{D}$ changes. In fact $\mathrm{DNS}$ for $\mathrm{P} / \mathrm{D}=1.05$ implies a much delayed transition to fully turbulent flow.

Wall shear stress distribution As for an infinite triangular pin array of $\mathrm{P} / \mathrm{D}=1.2$, as $\mathrm{Re}_{\text {bulk }}$ increases from 6, 000 to 24,000, a peak location of the wall shear stress moves from $\phi=25^{\circ}$ (Cases A1, B1) to $20^{\circ}$ (Case D), and the distribution becomes flatter as shown in Figure 4, where DNS for $\operatorname{Re}_{\text {bulk }}=9,120$ shows a much peaky trend in comparison with DNS $\operatorname{Re}_{\text {bulk }}=24,300$. Also in the figure, several higher $\mathrm{Re}$ cases are plotted for comparisons from results of $k-\varepsilon$ nonlinear ${ }^{9}$. In fact it has been reported by Trupp et al., ${ }^{8)}$ that in their experiments of much higher $\mathrm{Re}_{\text {bulk }}$ region, the wall shear stress distributions were shown to hardly vary from the experimental plots shown in Figure $4\left(\operatorname{Re}_{b}=24,300\right)$. Also Hooper ${ }^{10)}$ reported the similar experimental results for a square rod array and suggested that the shape of the wall shear stress distributions was almost independent of the Reynolds number in the highly turbulent flow regime. The DNS calculation results are compared and found in agreement with this trend in high Reynolds number regime (case $\mathrm{D}$ for $\mathrm{Re}_{\mathrm{b}}=24,300$ ). They indicate that the flattening is considered to be due to the increase in the secondary flow intensity with increase in $\mathrm{Re}_{\text {bulk }}$. It is noted here, however, that the $\mathrm{k}-\varepsilon$ nonlinear model rendered no stable solutions for flows with $\mathrm{Re}_{\text {bulk }}$ lower than $\sim 20,000$.

Secondary flows Figure 5 shows the calculated secondary flow distributions in a minimum unit flow cell after the time-average of instantaneous flow distributions in all the 12 unit cells $\left(\mathrm{P} / \mathrm{D}=1.2\right.$ for $\mathrm{Re}_{\tau}$ $=400$ and 1,400), where the vortex center is shown also to shift to the narrower gap region in highly turbulent flows. A maximum amplitude of the secondary flows is $0.6 \%$ and $0.7 \%$ of the bulk flow velocity for $\mathrm{Re}_{\tau}=400$ and 1,400, respectively.

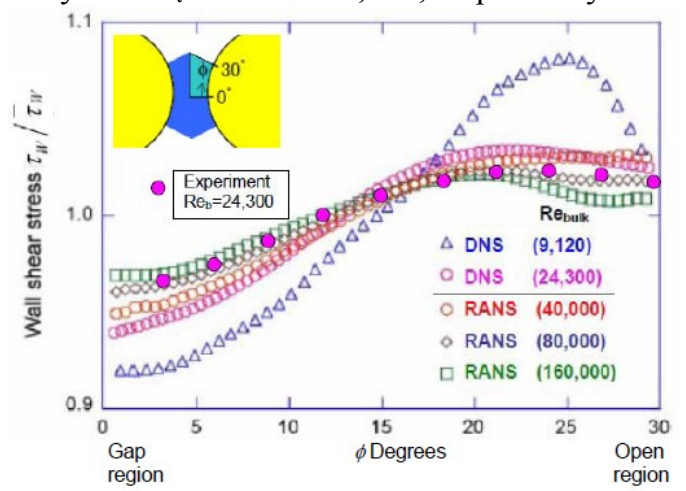

Figure 4. Wall shear stress distribution for various Reynolds numbers $(\mathrm{P} / \mathrm{D}=1.2)$

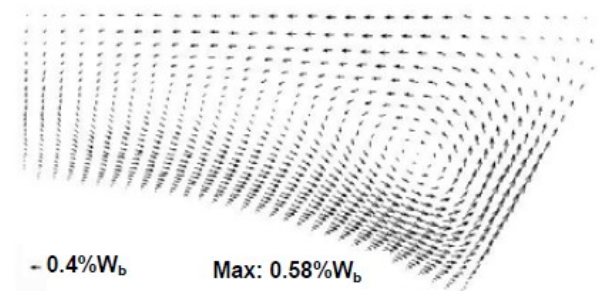

(a) $\mathrm{Re}_{\tau}=400\left(\mathrm{Re}_{\text {bull }} \cong 6,000\right)$

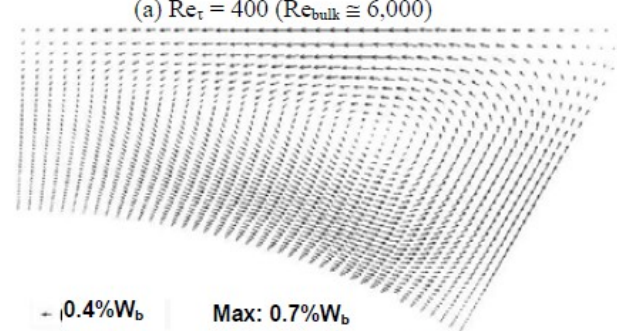

(b) $\operatorname{Re}_{\tau}=1,400\left(\operatorname{Re}_{\text {bull }} \cong 23,800\right)$

Figure 5. Secondary flow distributions $(\mathrm{P} / \mathrm{D}=1.2) ; \mathrm{w}_{\mathrm{b}}$ is the bulk velocity in the main flow direction. 


\subsubsection{Influence of pitch to diameter ratio on turbulent flow fields}

In comparison with the result for $\mathrm{P} / \mathrm{D}=1.2$, differences in the axial velocity profile for $\mathrm{P} / \mathrm{D}=1.1$ and 1.05 with $\operatorname{Re}_{\tau}=400$ and 600 extend between those at center region and gap region as shown in Figure 6 . It is caused by the increase in relative flow area of the center region (toward $\phi=30^{\circ}$ ) and the decrease in gap region (toward $\phi=0^{\circ}$ ) as $\mathrm{P} / \mathrm{D}$ decreases. Figure 7 shows the wall shear stress variations for $\mathrm{P} / \mathrm{D}=1.2,1.1$, and 1.05 for $\mathrm{Re}_{\tau}=400$. The wall shear stress distribution varies over wider range with the decrease in $\mathrm{P} / \mathrm{D}$.
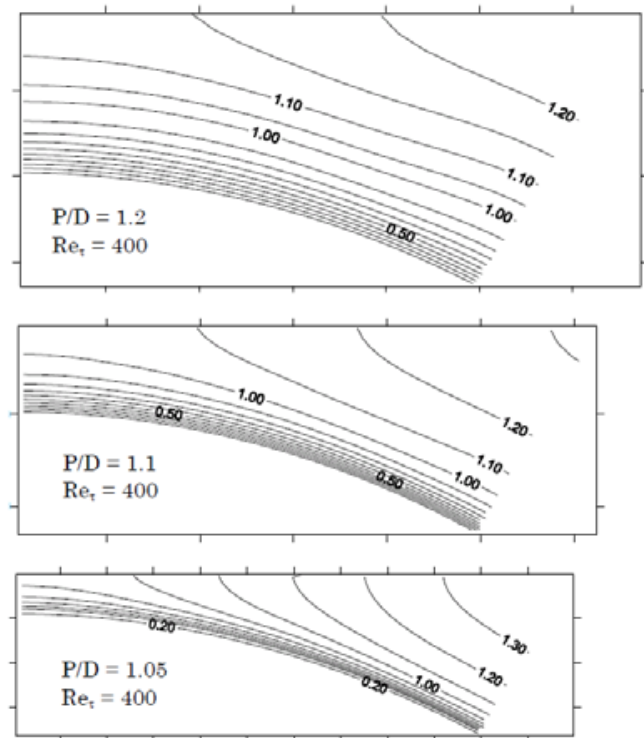

Figure 6. Axial velocity profile $\left(\mathrm{Re}_{\tau}=400, \mathrm{P} / \mathrm{D}=1.2\right.$, 1.1 , and 1.05 )

In general the secondary flow vorticity concentrates in the vicinity of the pin wall as $P / D$ decreases, and the turbulence anisotropy is enhanced in the gap region of the tight lattice subassemblies. Also it has been shown that the present numerical method could provide good physical insights into Reynolds stress including the behaviors of its azimuthal components in the gap region of a tight lattice subassembly.

It is noted that these DNS calculations have suggested a symptom of flow pulsation in the gap region for the tightly spaced $\mathrm{P} / \mathrm{D}=1.05$ configuration. To delineate better, it is noted that much longer axial span of the flow channels must be covered. Next Section and Section 3.2 will provide more complete description of the phenomena.

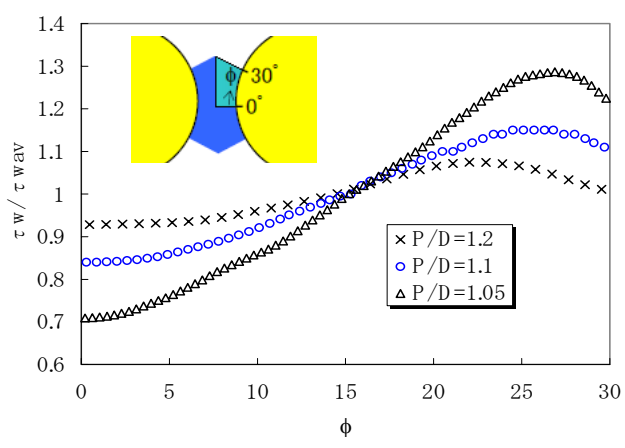

Figure 7. Wall shear stress distributions $\left(\operatorname{Re}_{\tau}=400\right.$, $\mathrm{P} / \mathrm{D}=1.2,1.1$, and 1.05$)$

\subsection{URANS Calculation of the Pulsating Flows in a Tight-Lattice Subassembly}

Axial coolant flow inside a tightly packed pin subassembly presents a complex behavior; experimental analysis had clearly shown that when reducing the pitch-to-diameter ratio $(\mathrm{P} / \mathrm{D})$ the turbulence field in pin subassemblies deviates significantly from that in a circular tube. Moreover for extremely tight configurations the existence of largescale periodic flow oscillations has been shown, which is responsible for the high inter-subchannel heat and momentum exchanges ${ }^{11)}$. Complete understanding of these oscillations has still to be achieved; the evidence shown to this point suggests that the oscillations are connected to interactions between eddy structures of turbulent flows in adjacent subchannels ${ }^{12)}$.

The aim of this Section is to show that the adoption of an Unsteady Reynolds Averaged Navier Stokes (URANS) methodology, instead of DNS which requires prohibitively huge computing resources for the simulation, allows us to reproduce these oscillations in the numerical model and to obtain accurate prediction of the averaged statistics.

The substantial failure of traditional steady state RANS modeling in the simulation of tight fuel bundles is not surprising. Beside turbulence itself (the actual flow field in a fuel pin subassembly is neither steady nor stable), the flow in a subassembly presents long-term, large scale coherent patterns in the direction of homogeneous turbulence. The contribution of these structures cannot be taken into account by a steady state simulation, unless specific ad-hoc models are introduced in the momentum equations. An ad-hoc model would lack generality, and would be geometry dependent (i.e. the same model would probably not work both on triangular arrays and quadratic arrays of pins), therefore its use is disputable. However these models are not available and a fully transient simulation is at the moment necessary.

In the present simulation the focus will be on the experimental setup of Krauss and Meyer ${ }^{13)}$. The choice is motivated by the detailed velocity distribution published and an extension of the 
experimental model that allows us to assume the flow as fully-developed. In particular we refer the flow characterized by Reynolds number of 38,754; hydraulic diameter of $33.5 \mathrm{~mm}$; P/D of 1.06; and water as a working fluid.

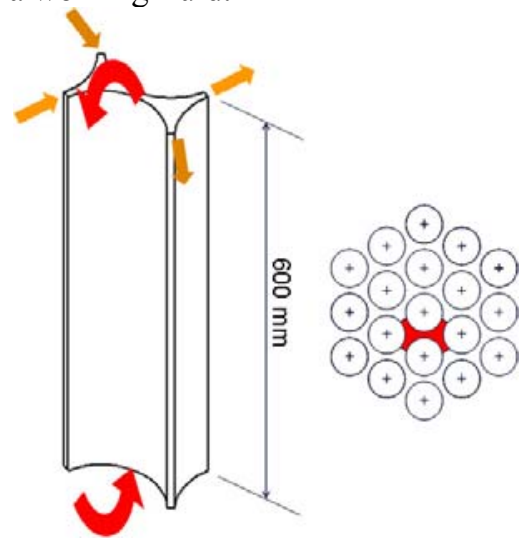

Figure 8 . Geometry and boundary conditions

A first step of the numerical approach to the long axial length phenomena is to make a choice of the length $\mathrm{L}$ by the averaged value of the dominant wave-number obtained from the experiment. A periodic boundary condition is imposed at the bottom and top of the flow channel (see Figure 8). Knowing that this approach is not general, we justify it by stating that this is to assess the ability of the methodology to reproduce critical averaged quantities (that are not in agreement with the experiment for steady state calculations).

The calculations presented in this work have been performed with a modified anisotropic k- $\varepsilon$ model $[5,8]$ in the CFD-code STAR-CD $3.26^{14)}$. We solve the boundary layer and for this the grid strategy includes: the first near wall mesh has been kept at a value of $\mathrm{y}^{+}=1$; in the wall layer, wall-normal cells have been used; and the sensitivity to the grid sizes has been tested out for steady state calculations. A total of more than 600,000 meshes have been used and the CFL condition has been kept below 0.2.

Results from two turbulence models, i.e., the modified anisotropic $\mathrm{k}-\varepsilon$ model $^{5,8)}$ and standard $\mathrm{k}-\varepsilon$ model have been compared and the superiority of anisotropic modeling has been demonstrated. This has been also true for even in unsteady simulations for the wall shear stress, Reynolds stresses and stream-wise velocity distributions. A snapshot of the shape of the cross-flow velocity oscillations across the narrow gap is shown in Figure 9. They are present also on the periodic boundaries giving place to a truly threedimensional pulsation, confirming the assumption of this oscillation to be strongly correlated spatially in the entire subassembly. Detailed discussions of the limits of the approach from the view points of turbulence modeling and the specific computational model are described by Merzari, et al., ${ }^{15)}$.

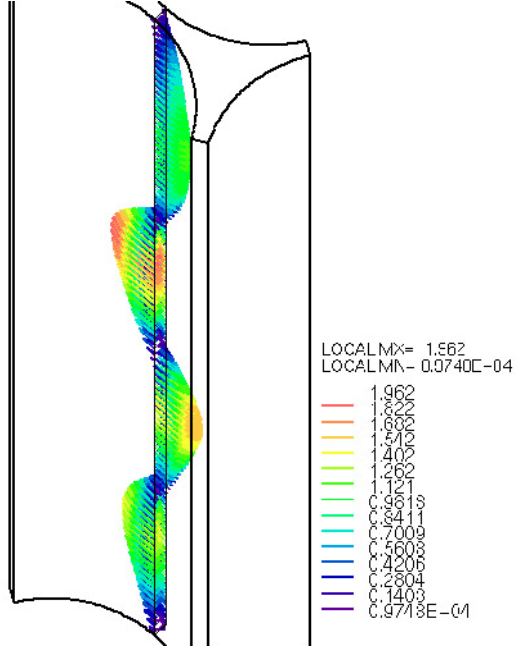

Figure 9. Flow oscillation between subchannels and cross flow velocity components

\section{Several Interesting Flow Phenomena in Non- Circular Channel Geometry Relevant to Fuel Pin Subassemblies}

In general, predicting detailed turbulent flow distributions (such as velocity, Reynolds stresses, RMS, ..) has been a challenge in thermal hydraulics analysis of fuel pin subassemblies. Here, we focus on the flows in simplified geometries, simplified but a good approximation to a subchannel of a fuel subassembly. Results obtained through DNS and LES codes are presented. Characteristic flow patterns will be analyzed and their similarity with the flow in the fuel pin subassemblies will be discussed. These computations are extremely expensive, for DNS, a case with higher highest Reynolds numbers $\left(\mathrm{Re}_{\mathrm{bulk}}=\right.$ 26,600) took 6 months to reach convergence on 128 CPUs on Earth Simulator ${ }^{1)}$. The LES simulations are overall less expensive, for a higher Reynolds number case, it has taken several weeks to reach convergence on another super computer available in Tokyo Institute of Technology (TSUBAME) with 32 CPUs.

\subsection{DNS and LES for Turbulent Flows in Eccentric Annulus Channels}

Both DNS and LES calculations presented in this section are based on the same numerical algorithms described in 2.1.1. In the case of LES, a dynamic model designed for boundary fitted coordinates has been employed. Fully developed turbulent flows have been simulated in two types of flow channel: concentric and eccentric annuli. DNS benchmark data is available for concentric configuration $^{16)}$, while some experimental results are available for eccentric annulus channels ${ }^{17)}$.

The ratio of inner circular column radius $R_{\text {in }}$ to outer pipe radius $R_{\text {out }}$ is 0.1 for the concentric annular channel and 0.5 for the eccentric annular channel. The eccentricity $\mathrm{e}=l /\left(\mathrm{R}_{\text {out }}-\mathrm{R}_{\mathrm{in}}\right)$ is 0.5 where $l$ is the 
distance between centers of two circles of the eccentric annulus channel. The length of the computational domain $\mathrm{L}$ is $6.28 \mathrm{xD}_{\mathrm{h}}$ where $\mathrm{D}_{\mathrm{h}}$ is the equivalent hydraulic diameter. This length is selected to be long enough to ensure that the fluctuating velocity components in the main flow direction are not correlated at two separated points with the distance of at least L/2. An a posteriori test has verified that the condition is met for the concentric case and most of the eccentric cases, with one exception that will be discussed in the following.

Periodic boundary conditions for velocity components are applied in the axial direction whereas a mean axial pressure gradient is an input to the LES/DNS calculations. DNS has been carried out for the flows in the eccentric annuli with the bulk Reynolds numbers 12,100 and 26,600 with $384 \times 128 \times 336$ and $768 \times 300 \times 768$ (circumferential $\times$ radial $\mathrm{x}$ axial), respectively. LES has been carried out for the bulk Reynolds numbers 3,200, 12,200 and 27,100 . The numbers of grid points used for LES

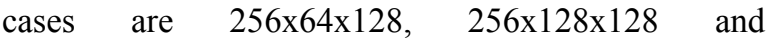
$512 \times 300 \times 512$. It is noted that additional simulations are made at different grid resolutions to test the dependence of the result on grid sensitivity.

\subsubsection{Verification of the DNS/LES method}

To test the code, a DNS of the flow in a concentric channel $(\mathrm{e}=0)$ has been carried out at $\mathrm{Re}=8,900$ (at two grid resolutions showing similar results: $256 \times 100 \times 336$ and $256 \times 64 \times 192$ ). Note that in the context that follows, $\mathrm{Re}$ refers to the bulk Reynolds number. Excellent agreement is obtained among the results of the DNS and LES, and the benchmark given by the spectral method by Chung, et. $a l .,{ }^{16)}$ for the averaged distribution of streamwise velocity, RMS of the velocity components and shear stress in the concentric annulus channel. Because DNS and LES have yielded exactly identical results, only the LES results are plotted in Figure 10 in comparison with the benchmark solution. This figure gives us confidence on the solidity of the numerical approach. In Figure $10, \mathrm{~W}_{\mathrm{b}}$ is the bulk velocity; $u_{\phi}$ is the azimuthal component of the flow velocity; and $w_{\tau}$ is the friction velocity.

Both DNS and LES simulations for the eccentric annulus show good agreement with the data of Nouri [17] for the case at Reynolds number equal to 26,600 , and excellent agreement with the DNS of Nikitin $^{18)}$ at $\mathrm{Re}=3,200$.

\subsubsection{Local laminarization and secondary flows}

Eccentric annular channels represent an ideal model for investigating inhomogeneous turbulent flows, where conditions of turbulence production vary significantly within the cross-section. For instance, the wall shear stress and the local Reynolds numbers are functions of the angular position. An interesting effect of this dependence is that the flow in the narrow gap appears to be subject to local laminarization, for appropriate values of the eccentricity and Reynolds number. Figure 11 shows the development of the stream-wise velocity profile calculated by LES with varying Reynolds number. It is worth noting here that these LES results show good agreement with DNS and experimental results for the corresponding Reynolds numbers (for the streamwise velocity and the RMS of the streamwise velocity component).

All the results obtained in this work and those by Gosset, et. al., ${ }^{19)}$ indicate that the transition to turbulence in the narrow gap is sensibly delayed if compared to the outer gap. An example is given by the case at $\mathrm{Re}=3,200$; while the stream-wise velocity profile in the wider gap appears to be in good agreement with profile of plane channel flow (as shown by Nikitin ${ }^{18)}$ for a similar case), in the narrow gap the velocity profile agrees very well with the laminar solution given by Snyder ${ }^{20)}$. As the Reynolds number grows the velocity field develops into a transitional state and slowly approaches fully developed turbulence. At $\mathrm{Re}=26,600$, the stream-wise velocity profile in the narrow gap still presents values sensibly higher than the log-law. Moreover, the viscous shear stress is characterized by unusually high values in the narrow-gap region, hinting again a transitional state.

Generally, higher eccentricity appears to have a stabilizing effect for laminar flows (see Gosset ${ }^{19)}$ for a geometry that share some similarities), delaying the transition to turbulence. At $\mathrm{Re}=3,200$, even if the stream-wise profile is laminar in the narrow gap, the RMS of the cross-velocity components is comparable to that in the wider gap, implying that turbulence is transported from the wider region to the narrow gap by the secondary flows. Moreover, in the narrow gap, RMS of the stream-wise velocity is sensibly smaller than that of the cross velocity components, an additional indication that the turbulence is not produced locally.

\subsubsection{Flow pulsation}

Observing the value of the cross velocity, we find that the flow field in the narrow gap shows an oscillating behavior characterized by low wavenumbers in the stream-wise direction. This oscillatory behavior is connected to pair of large scale vortex located near the border of the narrow gap, which resembles closely the structure of the flow in tight fuel pin assemblies and qualify the eccentric channel as the prototype geometry to study this kind of phenomena. When Reynolds averaging is performed the flow field exhibit secondary flows in the cross section.

The presence of strong oscillations in the case at $\mathrm{Re}=3,200$ increases the value of the two point correlation factor and therefore suggests us to employ a larger integral length-scale in the axial direction. Calculated velocity fluctuations at a distance of $\mathrm{L} / 2$ are strongly correlated, and therefore the quality of this simulation is disputable. Moreover, if simulation of the oscillations is to be pursued, particular care 
must be taken for the wave-number spectra to be reproduced accurately. However, in an attempt of this kind of simulation for the first time, it was considered sufficient to meet our objective to point out an existence of coherent structure even in this geometry.

As the Reynolds number increases, the oscillations at low wave-number become less dominant, and at $\mathrm{Re}=26,600$, they are not significant anymore. Figure 12 shows a three-dimensional plot of the instantaneous cross velocity component in the narrow gap $P 3$ as a function of the streamwise coordinate and the relative distance from the outer wall (the figure for $R e=12,100$ is plotted on the same frame and is separated by 2 units in the y-direction for a comparison). There, $\mathrm{y}=0$ and $\mathrm{y}=1$ correspond to the outer and inner wall positions along the $P 3$ line, respectively. It is noted that the transition to full turbulence finally occurs in the narrow gap after $\mathrm{Re}=3,200$ since the profile at $\mathrm{Re}=12,100$ presents a complex wave number spectrum. The low wave number component is far less dominant if compared to the case at $R e=3,200$. Other evidence not presented here also implies a decreasing intensity of the turbulent structure as Reynolds number increases. A possible cause might be a strong interaction and energy transfer between coherent modes (causing the oscillations) and incoherent modes (related to the chaotic patterns of turbulence). If that is the case, the local phenomenon of the transition to turbulence might be triggered by the coherent structures.

For higher values of eccentricity it appears that, from investigations currently under development (at an eccentricity equal to 0.95 ), the oscillations might be still dominant at higher values of the Reynolds number and even when the flow in the narrow gap is fully developed. This behavior would show strong similarities with the behavior of the flow in pin assemblies, where the subsistence of the oscillations is observed only above a threshold of the P/D ratio.

\subsubsection{Summary of the flow behaviors in eccentric annuli channels}

It is our inference that the delay in laminar to turbulent transition in the narrow gap region leads to a large scale oscillation in the process of turbulent flow exchanges between almost-laminar region (narrow gap) of the low-energy and the high-energy region (larger gap) of the eccentric annulus channel. This large scale oscillation transports turbulence from the wide open region to the narrow gap. It is noted that the coherent structure observed at the low Reynolds numbers bears a striking resemblance to those observed in the shear-free mixing layer. Furthermore, at higher Reynolds numbers, it has been found that these oscillations die out (or become less significant) for the eccentricity and diameters considered in this work. Larger eccentricity cases would lead to a further delay in the transition. It is, therefore, future work to find a correlation among the eccentricity and Reynolds number for the onset of stable oscillations. Also in the limited computational domain, in particular, in the stream-wise direction with only $\mathrm{L}=6.28 \mathrm{x} D h$, it may be premature to discuss the intermittent behaviors of the large scale oscillations based on the calculational results illustrated in this section. Therefore, as a preliminary step of the investigation, the large scale (global) oscillation phenomena are investigated for flows in a channel of simplified geometry with a help of LES in the next Section 3.2.
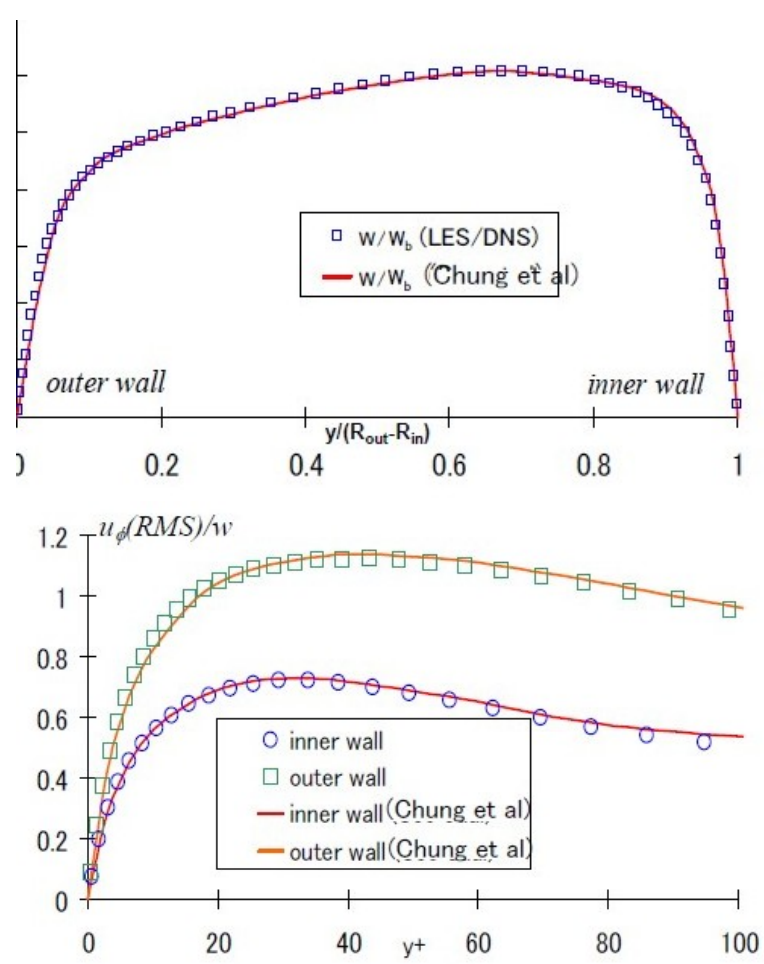

Figure 10. Stream-wise velocity and RMS profiles by LES in a concentric annular channel

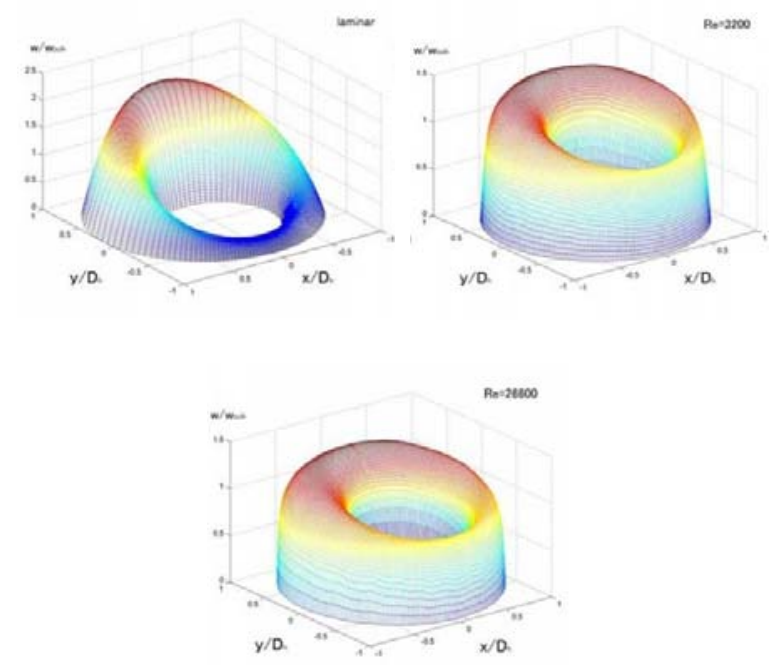

Figure 11. Stream-wise velocity profiles by LES for different Reynolds numbers 


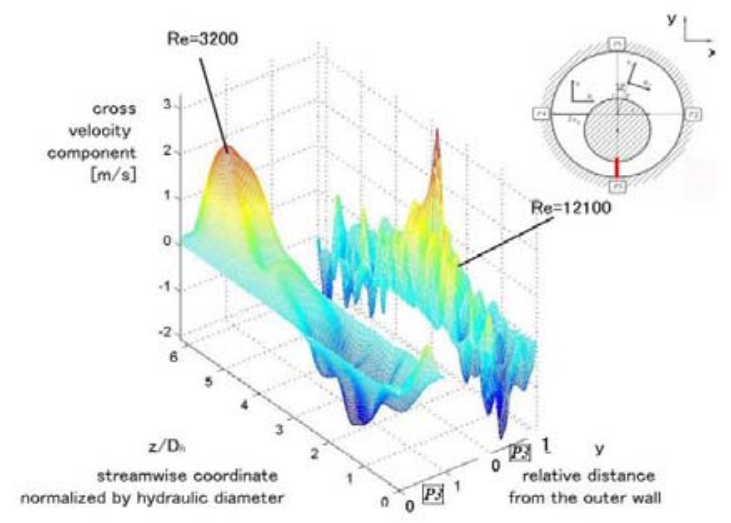

Figure 12. Cross flow velocity distribution at the narrow gap $P 3$ at two Reynolds number cases $(\mathrm{Re}=$ $3,200$ and 12,100$)$

\subsection{LES for Pulsating Flows in Inter-Connected Two Rectangular Ducts with a Narrow Gap}

Several other simplified geometries have been studied over the years by several investigators e.g., Meyer $^{21)}$, Biemuller ${ }^{22)}$, Lexmond ${ }^{12)}$ and Guellouz [23]. The focus here will be on the characterization of the axial flow in two rectangular ducts inter-connected by a narrow gap. The choice is motivated by the availability of detailed flow distributions at a relatively low Reynolds number. For this geometry, like for the eccentric annulus, turbulent structure is observed on the border of the gap, and the flow in the gap presents a strong oscillatory behavior. The simulation has been carried out by LES, with the traditional Smagorinsky model. Periodic boundary conditions in the stream-wise direction and non-slip wall boundary conditions have been employed. Due to computational limitations, the domain in the streamwise direction has been chosen to contain exactly two structures; i.e., the wavelength of the oscillations has therefore been forced to match the experimental value. The frequency spectrums of the oscillations as well as the averaged stream-wise velocity have been found in good agreement with the measurement.

The principal aim of this simulation was to shed some light on the physics of the oscillations. In order to do so, a large database of snapshots of the turbulent flow field has been collected. A statistical tool known as POD (Proper Ortho-normal Decomposition $^{24)}$ ) has been employed to decompose the flow into a set of ortho-normal modes, by a variational principle of energy maximization. Sections of the most energetic mode are showed in Figs. 13 and 14. Figure 13 is a vector plot of the velocity in the gap mid-plane. Three sets of vortexes are recognizable in the gap and on the edges of the gap. Figure 14 shows three-dimensional plots of the cross velocity for the same eigen-mode. All velocities are expressed in $\mathrm{m} / \mathrm{s}$ while the spatial coordinates are given in centimeters.

It appears that the most energetic mode responsible for the mass transfer between rectangular channels is characterized by high values of the cross velocity in the gap region, due to the coupling between eddy structures in the two channels through a third set of vortexes located in the middle of the gap. After performing the POD, it has been found that most of the energy is accumulated by a very limited set of modes, dominated by a single wave-number. This raises an issue of consistency.

Ultimately, what is essential for a successful application of POD on numerical data is for the computation itself to be representative of the dynamics of the real system. Thus, if the numerical calculation is dominated by a discrete set of modes as in the present case, it is important to verify whether this set is a good approximation to the continuous wave-number spectra of the experiment, since a great part of the dynamics of the system can be represented by the evolution of these modes. A more extensive discussion on the peculiarities of the numerical simulation of flow oscillations has therefore to be assessed.

It is worth while to note here that, when a quasi-periodic phenomenon is simulated in the streamwise direction using the periodic boundary conditions, it should be ensured that the computational grid is able to reproduce the range of wave lengths present in the simulation. Large scale structure involves small wave numbers, usually close to the limit of the wavenumber resolution of a given grid, i.e., $\Delta k=2 \pi / L$.

As a consequence, whether a simulation will be representative of the system can be determined $a$ priori only if the domain length is sufficiently long ensuring the high wavelength resolution or if experimental data is available that supports the choice of the length of the computational domain.

However, provided that the grid and the numerical practices involved are adequate, POD could be successfully applied on the results of the computation to gain additional insights into the physics of the oscillations. For instance, POD can be used in association with Galerkin projection to reduce the Navier-Stokes equations to a set of non-linear ODEs. This approach, experimented with success on wall turbulence, is promising for the case of the oscillations. It provides simplified models for subchannel analysis and clarifies the mechanisms lying behind the oscillations.

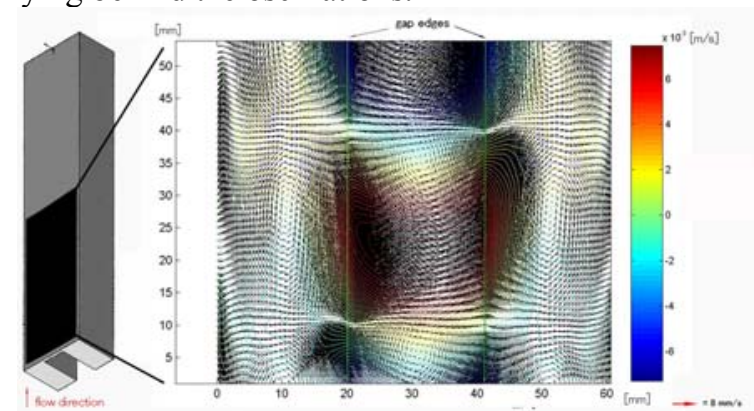

Figure 13. Principal mode of the POD decomposition of the flow on the plane across the narrow gap region, horizontally extended toward the two main channel regions 


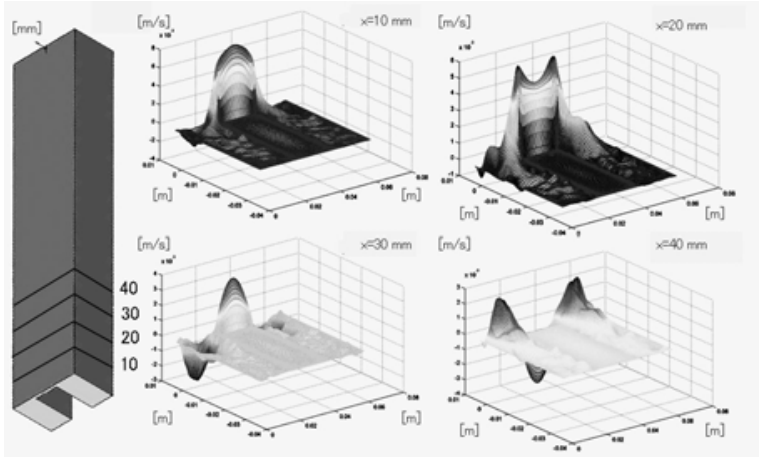

Figure 14. Principal mode of the POD decomposition of the flow, 3D plots of the cross velocity at different cross sections of the streamwise coordinate at $\mathrm{x}=10$, 20,30 and $40 \mathrm{~mm}$

\section{Concluding Remarks}

We have illustrated the turbulence phenomena that are peculiar to the flows in tight lattice nuclear fuel subassemblies, eccentric annuli and rectangular channels interconnected by a narrow gap. These flows are considered to share more or less the similar turbulence structure and behaviors, and commonly characterized by anisotropy and exhibit turbulencedriven secondary flows in non-circular channels, local transitions between laminar and turbulent flows at a narrow gap, and global pulsations leading to the coolant mixing between two subchannels or across the narrow gap.

At present, the mesh grid requirement makes DNS computations almost impossible for fully turbulent flow of high Reynolds numbers in a subchannel even on any high-end supercomputers available. In this paper, therefore, a DNS attempt has been limited to flows of lower Reynolds number, i.e., $\operatorname{Re}<24,000$ with the assumption of an infinite array of smooth pin bundle.

For flows in a subchannel of a triangular pin array subassembly, a key to reliable results is to predict precisely a distribution of turbulence-driven secondary flows. As a result, for a fuel pin pitch to diameter ratio $\mathrm{P} / \mathrm{D}=1.2$, it has been revealed that calculated profiles of the main flow velocity, wall shear stress, Reynolds stress, ... , etc. vary with decreasing $\mathrm{Re}$, while it is implied that they would become almost independent of $\mathrm{Re}$ in the highly turbulent flow regime. This is in agreement with the experimental data available.

It is found that the flow is fully turbulent at almost everywhere in the subchannel at $\operatorname{Re} \sim 24,000$ for a pin subassembly with $\mathrm{P} / \mathrm{D}=1.2$. It has been shown numerically that a local laminar transition starts to develop in the gap region at $\operatorname{Re} \sim 20,000$. This local laminarization is enhanced as $\mathrm{P} / \mathrm{D}$ decreases and the fully transition to turbulent flow will be delayed. These local laminar-turbulent transition phenomena have been also investigated by LES as well as by DNS for turbulent flows in eccentric annuli channels. The results have confirmed the similarity to the phenomena taking place in the subchannel flows in a fuel subassembly.

Among many approaches of CFD, DNS is the most preferred when we try to investigate the phenomena whose mechanisms are unknown or not clearly understood. However, because of the requirement for prohibitively huge computing resources, DNS cannot always readily be carried out. RANS is useful only when mechanisms of phenomena are well known and understood. Overall, in these respects, LES would be a good choice as illustrated in the last two sections in this paper. There LES has reproduced almost identical results with DNS for the turbulent flows in an eccentric annulus. It has provided physical insights into the global flow pulsation phenomena in the inter-connected subchannel flow systems.

As the simulation-based design methods would largely be benefited by the progress of CFD, the more increased use of the CFD is expected. It is, however, always reminded that CFD is not almighty and its relative position to experiments remains to be complementary. In particular, when such an approach as RANS that requires turbulence models is employed in the simulation, users must be alerted that calculational results are totally dependent on the modeling and always within a realm of only known mechanisms on which basis the turbulence models are constructed.

Finally, since the CFD provides us much more detailed information than experiment, importance of how we extract physics out of a huge body of computed data cannot be overemphasized. As an example of the method, such a numerical technique as Proper Ortho-normal Decomposition has been shown to be very useful.

\section{References}

1. http://www.es.jamstec.go.jp/index.en.html

2. H. Ninokata, A. Efthimiadis and N. E. Todreas, Distributed Resistance Modeling of WireWrapped Rod Bundles, Nucl Eng Des, 104, 93 102, 1987.

3. C. W. Rapley and A.D. Gosman, A.D., The Prediction of Fully Developed Axial Turbulent Flow in Rod Bundles, Nucl Eng Des, 97, 313-325, 1986.

4. K. B. Lee and H. C. Jang, A Numerical Prediction On The Turbulent Flow In Closely Spaced Bare Rod Arrays by a Nunlinear k-e Model, Nucl Eng Des, 172, 351-357, 1997.

5. E. Baglietto and H. Ninokata, CFD Modeling of Secondary Flows in Fuel Rod Bundles, N6P363, $6^{\text {th }}$ Int. Top. Mtg on Nuclear Reactor Thermal Hydraulics, Operations and Safety (NUTHOS-6), Nara, 2004.

6. Y. Zhang, R. L. Street and J. R. Koseff, A NonStaggered Grid, Fractional Step Method for TimeDependent Incompressible Navier-Stokes 
Equation in Curvilinear Coordinates, J. Comput. Phys., 114, 18-33, 1994.

7. T. Misawa and H. Ninokata, Calculation of Detailed Velocity Distributions in Fuel Pin Bundles of Infinite Triangular Array Configuration Using Direct Numerical Simulation, $6^{\text {th }}$ Int. Top. Mtg on Nuclear Reactor Thermal Hydraulics, Operations and Safety (NUTHOS-6), Nara, 2004.

8. A. C. Trupp and R. A. Azad, The Structure of Turbulent of Flow in Triangular Array Rod Bundles, Nucl Eng Des, 32, 47-84, 1975.

9. E. Baglietto and H. Ninokata, A Turbulence Model Study for Simulating Tight Lattice Rod Bundles, Nucl Eng Des, 235, 773-784, 2005.

10. J. D. Hooper, Developed Single Phase Turbulent Flow Through a Square-Pitch Rod Cluster, $\mathrm{Nucl}$ Eng Des, 60, 365-379, 1980.

11. S.V. Moller, On Phenomena of Turbulent Flow Through Rod Bundles, Experimental Thermal and Fluid Science, 4, 25-35, 1991.

12. M. Lexmond and T. Van der Haagen, Visualization of the Vortex Street and Characterization of the Cross Flow in the Gap Between two Subchannels, $11^{\text {th }}$ NURETH (paper:122), 2005.

13. T. Krauss and L. Meyer, Experimental Investigation of Turbulent Transport of Momentum and Energy in a Heated rod Bundle, Nucl Eng Des, 18, 185-206, 1998.

14. STAR-CD Documentation, version 3.26

15. E. Merzari and H. Ninokata, Unsteady Reynolds Averaged Navier-Stokes Simulation for an Accurate Prediction of the Flow Inside Tight Rod Bundles, $12^{\text {th }}$ NURETH, Pittsburgh, to be presented (2007).
16. S. Y. Chung, G. H. Rhee and H. J. Sung, Direct Numerical Simulation of Turbulent Concentric Annular Pipe Flow. Part 1: Flow field, Int $J$ of Heat and Fluid Flow, 23, 426-440, 2002.

17. J. M. Nouri, H. Umur and J. H. Whitelaw, Flow of Newtonian and non-Newtonian Fluids in Concentric and Eccentric Annuli, J. Fluid Mech. 253, 617-641, 1993.

18. N. V. Nikitin, Direct Numerical Simulation of Turbulent Flows in Eccentric Pipes, Computational Mathematics and Mathematical Physics, 46, 509-526, 2006.

19. A. Gosset and S. Tavoularis, Laminar Flow Instability in a Rectangular Channel with a Cylindrical Core, Phys. Fluids, 18, 2006.

20. W. T. Snyder and G. A. Goldstein, An Analysis of Fully Developed Laminar Flow in an Eccentric Annulus, A.I.Ch.E. Journal, 11, 462-467, 1965.

21. L. Meyer and K. Rehme, Large-Scale Turbulence Phenomena in Compound Rectangular Channels, Experimental Thermal and Fluid Science, 8, 286304, 1994.

22. M. Biemuller, L. Meyer and K. Rehme, Large Eddy simulation and Measurement of the Structure of Turbulence in two Rectangular Channels Connected by a Gap, Engineering Turbulence Modelling and experiments, 3, 249258, 1996.

23. M. S. Guellouz and S. Tavoularis, The Structure of Turbulent Flow in a Rectangular Channel Containing a Cylindrical Rod - Part 1: Reynolds Averaged Experiments, Experimental Thermal and Fluid Science, 29, 59-73, 2000.

24. J. L. Lumley, Stochastic tools in turbulence, Academic University Press (1970).

Table 1. Calculation conditions of the simulation cases

\begin{tabular}{|c|c|c|c|c|c|c|c|c|}
\hline Case & $\mathrm{Re}_{\tau}$ & $\mathrm{P} / \mathrm{D}$ & $N_{\phi} \times N_{r} \times N_{z}$ & $\mathrm{r}^{+} \Delta \phi$ & $\Delta \mathrm{r}^{+}$ & $\Delta \mathrm{z}^{+}$ & $\Delta \mathrm{t}^{+}$ & Bulk $R e_{b}$ \\
\hline A1 & \multirow{3}{*}{400} & 1.2 & $384 \times 28 \times 128$ & $6.2 \sim 7.3$ & $1.0 \sim 12.8$ & 10.0 & $1.5 \mathrm{E}-4$ & 5,972 \\
\hline A2 & & 1.1 & $624 \times 34 \times 128$ & $6.1 \sim 7.3$ & $1.0 \sim 12.8$ & 10.0 & $1.5 \mathrm{E}-4$ & 5,969 \\
\hline A3 & & 1.05 & $864 \times 41 \times 128$ & $6.5 \sim 7.8$ & $1.0 \sim 12.8$ & 10.0 & $1.5 \mathrm{E}-4$ & 5,863 \\
\hline B1 & \multirow{3}{*}{600} & 1.2 & $576 \times 40 \times 160$ & $6.2 \sim 7.3$ & $1.0 \sim 13.7$ & 12.0 & $1.5 \mathrm{E}-4$ & 9,181 \\
\hline B2 & & 1.1 & $864 \times 50 \times 160$ & $6.6 \sim 7.9$ & $1.0 \sim 13.4$ & 12.0 & $1.5 \mathrm{E}-4$ & 9,384 \\
\hline B3 & & 1.05 & $1,152 \times 60 \times 160$ & $7.4 \sim 8.8$ & $1.0 \sim 13.5$ & 12.0 & $8.0 \mathrm{E}-5$ & 9,646 \\
\hline $\mathrm{C}$ & 1,000 & 1.2 & $912 \times 67 \times 256$ & $6.5 \sim 7.7$ & $1.0 \sim 16.8$ & 14.0 & $1.5 \mathrm{E}-4$ & 16,276 \\
\hline $\mathrm{D}$ & 1,400 & 1.2 & $1,056 \times 80 \times 320$ & 7.9 9.3 & $1.0 \sim 16.9$ & 14.0 & $1.2 \mathrm{E}-4$ & 23,763 \\
\hline
\end{tabular}

Note: $N_{\phi}, N_{r}$ and $N_{z}$ are the number of grid points in $\phi$ - and r-directions (see Figure 2(c)) and in the main flow direction $\mathrm{z}$, respectively. $\mathrm{r}^{+} \Delta \phi, \Delta \mathrm{r}^{+}, \Delta \mathrm{z}^{+}$and $\Delta \mathrm{t}^{+}$are given in the non-dimensional form by $r \Delta \phi \operatorname{Re}_{\tau} / D_{n}, \Delta r \operatorname{Re}_{*} / D_{n}, \Delta z \operatorname{Re}_{*} / D_{n}$ and $w_{i} \Delta t / D_{n}$, respectively. 